\title{
Linear Micropolar Elasticity Analysis of Stresses in Bones under Static Loads
}

\author{
V. A. Eremeyev, ${ }^{a, 1}$ A. Skrzat, ${ }^{\mathrm{b}, 2}$ and F. Stachowicz ${ }^{\mathrm{b}, 3}$ \\ a Gdansk University of Technology, Gdansk, Poland \\ b Rzeszów University of Technology, Rzeszów, Poland \\ 1 eremeyev.victor@gmail.com \\ 2 askrzat@prz.edu.pl \\ 3 stafel@prz.edu.pl
}

We discuss the finite element modeling of porous materials such as bones using the linear micropolar elasticity. In order to solve static boundary-value problems, we developed new finite elements, which capture the micropolar behavior of the material. Developed elements were implemented in the commercial software ABAQUS. The modeling of a femur bone with and without implant under various stages of healing is discussed in details.

Keywords: bone, implant, Cosserat continuum, micropolar elasticity, numerical simulation, finite element method.

Introduction. Bones constitute a particular class of materials which may demonstrate very complex behavior including such phenomena as growth, anisotropy, porosity, piezoelectricity, etc., see, e.g., [1-4]. Mechanics of porous materials is a quite developed branch of mechanics. It includes various models of material behavior. Among these models the continuum Cosserat called also micropolar medium plays an important role. For example, the micropolar elasticity may predict size-effect observed for bones. Within the micropolar elasticity, each material point possesses properties of a rigid body that is it has both translational and rotational degrees of freedom. The duals for the translational and rotational degrees of freedom are forces and couples (moments). Since the bone can be treated as a microstructure material with such micro-elements as struts modeled as beams, the appearance of moments seems to be quite natural for such materials. In fact, a bone can be considered as an example of an open-cell foam. Since in foam struts there exist moments in addition to forces, this naturally leads to the micropolar elasticity, see the original works of Lakes and coworkers [5-9], where few experiments on porous materials including bones are performed. Recently, the homogenization technique leading to the micropolar elasticity in the case of bones is presented in [10-14]. In particular, the latter papers give the material parameters of the micropolar elasticity. Let us also note that such microstructured materials as foams, porous media, beam lattices may lead to more general models of continuum mechanics, see for example, the strain gradient elasticity [15-21]. Let us note that for modeling of a bone, various mechanical models were proposed, for example, media with internal variables [22-25].

The micropolar theory may play an even more important role if considered from the fracture mechanics point of view. Here the so-called constraint pseudo continuum Cosserat is the most used model. First using this model one observes that the stress and moment singularity may be quite different in comparison with the classic fracture mechanics [26-28]. This cause a question what type of fracture criterion should be used to decide when the crack starts propagating and what material parameters should be evaluated to determine the material toughness. Two criteria were discussed in $[27,28]$ to tackle the problem: energy release rate criterion and the maximum stress criterion proposed. They both demonstrate similar ability in predicting the fracture while the second one looked 
more promising. The situation complicated even further when one considers an interface crack in such material [29]. Finally when analyzing a moving crack an additional problem of the stability of the crack propagating in such material arises [30-33]. Here various effects were determined and the aforementioned criteria were used to estimate material toughness. From the presented analysis, the fracture criterion based on the maximum stress again demonstrated better performance. However, all those results are still to be verified experimentally.

The aim of the paper is the implementation of developed finite elements into ABAQUS and analysis of the static deformations of the loaded bone with an implant. The paper is organized as follows. First, we recall the basic equations of the micropolar elasticity. Then we introduce new tetrahedral micropolar finite element. Here we follow previous results [34-36] for the implementation of the hexahedral finite micropolar element. Finally, within the micropolar elasticity, we consider the modeling of a femur bone with an implant using the micropolar finite elements.

Basic Equations of the Linear Isotropic Micropolar Elasticity. The micropolar continuum model was introduced by Cosserat brothers more than hundred years ago [37]. For the current state-of-the-art, we refer to [38, 39]. Unlike classic elasticity, within the micropolar elasticity, we introduce not only displacements $u_{j}$ but also rotations $\theta_{j}$ as kinematic variables. Here we adopt the following convention: Latin indices take values 1 , 2,3 , so $i, j, k=1,2,3$. The equilibrium equations take the form

$$
\begin{gathered}
\sigma_{j i, j}=0, \\
m_{j i, j}+e_{i j k} \sigma_{j i}=0,
\end{gathered}
$$

where $\sigma_{j i}$ and $m_{j i}$ are the stress and couple stress tensors, respectively, and $e_{i j k}$ is the Levi-Civita permutation symbol.

Hereinafter we use the Einstein summation rule over repeating indices and, for simplicity, we neglect body forces and couples. Equations (1) and (2) are the local balances of momentum and moment of momentum. The strain tensors in the micropolar elasticity are given by $[40,41]$

$$
\varepsilon_{i j}=u_{j, i}+e_{i j k} \theta_{k}, \quad \kappa_{i j}=\theta_{i, j} .
$$

Note that $\sigma_{j i}$ and $m_{j i}, \varepsilon_{i j}$ and $\kappa_{i j}$ are all nonsymmetric tensors, in general.

In what follows we are restricted ourselves considering isotropic micropolar materials $[38,42,43]$. Using the modified for the micropolar elasticity the Voigt notation [34-36] the constitutive relations transform into the following form:

$$
\left\{\sigma_{M}\right\}=[C]\left\{\varepsilon_{M}\right\}
$$

where

$$
\begin{gathered}
\left\{\sigma_{M}\right\}=\left\{\begin{array}{c}
\sigma \\
m
\end{array}\right\}, \quad\left\{\varepsilon_{M}\right\}=\left\{\begin{array}{c}
\varepsilon \\
\kappa
\end{array}\right\}, \\
\sigma=\left\{\sigma_{x x}, \sigma_{y y}, \sigma_{z z}, \sigma_{x y}, \sigma_{y x}, \sigma_{y z}, \sigma_{z y}, \sigma_{x z}, \sigma_{z x}\right\}^{T}, \\
m=\left\{m_{x x}, m_{y y}, m_{z z}, m_{x y}, m_{y x}, m_{y z}, m_{z y}, m_{x z}, m_{z x}\right\}^{T}, \\
\varepsilon=\left\{\varepsilon_{x x}, \varepsilon_{y y}, \varepsilon_{z z}, \varepsilon_{x y}, \varepsilon_{y x}, \varepsilon_{y z}, \varepsilon_{z y}, \varepsilon_{x z}, \varepsilon_{z x}\right\}^{T},
\end{gathered}
$$




$$
\kappa=\left\{\kappa_{x x}, \kappa_{y y}, \kappa_{z z}, \kappa_{x y}, \kappa_{y x}, \kappa_{y z}, \kappa_{z y}, \kappa_{x z}, \kappa_{z x}\right\}^{T},
$$

and stiffness matrix $[C]$ consists of the following submatrices [34-36]:

$$
[C]=\left[\begin{array}{ll}
A & 0 \\
0 & B
\end{array}\right],
$$

with

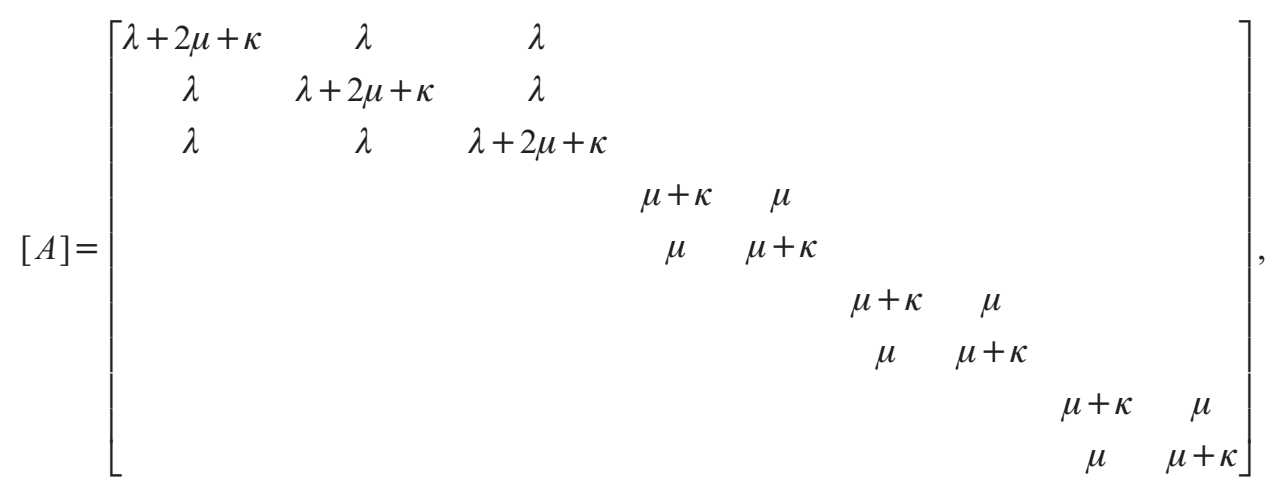

and matrix $B$ has a similar structure:

$$
[B]=\left[\begin{array}{ccccccccc}
\alpha+\beta+\gamma & \alpha & \alpha & & & & & \\
\alpha & \alpha+\beta+\gamma & \alpha & & & & & \\
\alpha & \alpha & \alpha+\beta+\gamma & & & & & & \\
& & & \gamma & \beta & & & \\
& & & & \beta & \gamma & & & \\
& & & & & \gamma & \beta & & \\
& & & & & \beta & \gamma & & \\
& & & & & & & \gamma & \beta \\
& & & & & & & \beta & \gamma
\end{array}\right] .
$$

Here $\lambda, \mu, \kappa$ and $\alpha, \beta, \gamma$ are material moduli. The latter ones depend on the engineering parameters as follows:

$$
\left\{\begin{array} { l } 
{ \kappa = \frac { 2 G N ^ { 2 } } { 1 - N ^ { 2 } } , } \\
{ \mu = G ( 1 - \frac { N ^ { 2 } } { 1 - N ^ { 2 } } ) , } \\
{ \lambda = \frac { 2 G v } { 1 - 2 v } , }
\end{array} \left\{\begin{array}{l}
\alpha=l_{t}^{2}(2 \mu+\kappa) \frac{1-\Psi}{\Psi}, \\
\beta=\left(l_{t}^{2}-2 l_{b}^{2}\right)(2 \mu+\kappa), \\
\gamma=2 l_{b}^{2}(2 \mu+\kappa) .
\end{array}\right.\right.
$$


$\mathrm{T}$ a b 1 e 1

Micropolar Material Data for Bones [5-9]

\begin{tabular}{||c|c|c||}
\hline Parameter & Symbol & Value \\
\hline Shear modulus (MPa) & $G$ & 4000 \\
\hline Poisson's ratio & $v$ & 0.25 \\
\hline Coupling number & $N$ & 0.5 \\
\hline Characteristic length (torsion) $(\mathrm{m})$ & $l_{t}$ & 0.00022 \\
\hline Characteristic length (bending) $(\mathrm{m})$ & $l_{b}$ & 0.00045 \\
\hline Polar ratio & $\Psi$ & 1.5 \\
\hline
\end{tabular}

The values of the engineering moduli for bones are given in Table 1.

4-Node Micropolar Tetrahedral Element. Following the technique used for developing of the 8-node hexahedral micropolar element $[35,36]$ the 4-node micropolar tetrahedral element has been developed. This element is suitable for domains of arbitrary (even very sophisticated) geometry, and, e.g., for bones. The position of an arbitrary point inside tetrahedron can be specified in Cartesian coordinates $\{x, y, z\}$ or in natural coordinates $\left\{\zeta_{1}, \zeta_{2}, \zeta_{3}, \zeta_{4}\right\}$. Natural coordinates are defined as the fraction of the volumes $V_{i}$ related to the volume $V$, where $V_{i}$ is defined as the volume of subtetrahedron bounded by the inside point and the face $F_{i}$ (Fig. 1). Natural coordinates are constrained by the equation:

$$
\zeta_{1}+\zeta_{2}+\zeta_{3}+\zeta_{4}=\frac{V_{1}}{V}+\frac{V_{2}}{V}+\frac{V_{3}}{V}+\frac{V_{4}}{V}=1
$$

So only three coordinates are independent. Mapping of natural coordinates into Cartesian ones is given as follows:

$$
\left\{\begin{array}{l}
1 \\
x \\
y \\
z
\end{array}\right\}=\left[\begin{array}{cccc}
1 & 1 & 1 & 1 \\
x_{1} & x_{2} & x_{3} & x_{4} \\
y_{1} & y_{2} & y_{3} & y_{4} \\
z_{1} & z_{2} & z_{3} & z_{4}
\end{array}\right]\left\{\begin{array}{l}
\zeta_{1} \\
\xi_{2} \\
\xi_{3} \\
\zeta_{4}
\end{array}\right\}=[J]\left\{\begin{array}{l}
\zeta_{1} \\
\xi_{2} \\
\zeta_{3} \\
\zeta_{4}
\end{array}\right\},
$$

where $x_{i}, y_{i}$, and $z_{i}$ are Cartesian coordinates of node $i$. The volume of tetrahedron takes the value

$$
V=\frac{1}{6} \operatorname{det}[J]
$$

From (15) it follows that

$$
\frac{\partial x}{\partial \xi_{i}}=x_{i}, \quad \frac{\partial y}{\partial \xi_{i}}=y_{i}, \quad \frac{\partial z}{\partial \xi_{i}}=z_{i}, \quad i=1,2,3,4 .
$$

The inverse of the Jacobian (which can be found numerically or in an explicit form) is

$$
[J]^{-1}=\frac{1}{6 V}\left[\begin{array}{llll}
\ldots & a_{1} & b_{1} & c_{1} \\
\ldots & a_{2} & b_{2} & c_{2} \\
\ldots & a_{3} & b_{3} & c_{3} \\
\ldots & a_{4} & b_{4} & c_{4}
\end{array}\right]
$$




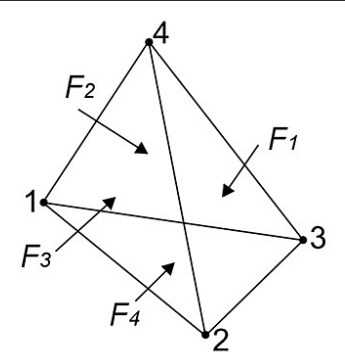

Fig. 1. Nodes and faces numbering.

The first column is omitted here because it is not important from the point of view of further derivation.

From (18) we obtain

$$
6 V \frac{\partial \xi_{i}}{\partial x}=a_{i}, \quad 6 V \frac{\partial \xi_{i}}{\partial y}=b_{i}, \quad 6 V \frac{\partial \xi_{i}}{\partial z}=c_{i}, \quad i=1,2,3,4 .
$$

Natural coordinates which are assumed to be the shape functions $\left(f_{i}=\zeta_{i}\right)$ are used in the interpolation of displacement field inside tetrahedron

$$
\left\{\begin{array}{l}
u \\
v \\
w
\end{array}\right\}=\left[\begin{array}{llll}
u_{1} & u_{2} & u_{3} & u_{4} \\
v_{1} & v_{2} & v_{3} & v_{4} \\
w_{1} & w_{2} & w_{3} & w_{4}
\end{array}\right]\left\{\begin{array}{l}
\xi_{1} \\
\xi_{2} \\
\zeta_{3} \\
\zeta_{4}
\end{array}\right\} .
$$

In order to find strains, we need displacements derivatives in terms of the Cartesian coordinates. Applying the chain rule to shape functions one obtains:

$$
\begin{aligned}
& f_{, x}=\frac{\partial f}{\partial \xi_{i}} \frac{\partial \zeta_{i}}{\partial x}=\frac{\partial f}{\partial \xi_{i}} \frac{a_{i}}{6 V}, \\
& f_{, y}=\frac{\partial f}{\partial \xi_{i}} \frac{\partial \zeta_{i}}{\partial y}=\frac{\partial f}{\partial \xi_{i}} \frac{b_{i}}{6 V}, \\
& f_{, z}=\frac{\partial f}{\partial \xi_{i}} \frac{\partial \zeta_{i}}{\partial z}=\frac{\partial f}{\partial \xi_{i}} \frac{c_{i}}{6 V} .
\end{aligned}
$$

Combining (18) and (21) and noticing that

$$
\frac{\partial f_{i}}{\partial \xi_{j}}=\left\{\begin{array}{lll}
1 & \text { for } & i=j \\
0 & \text { for } & i \neq j
\end{array}\right.
$$

the shape functions derivatives can be found from the inverse of the Jacobian

$$
[J]^{-1}=\frac{1}{6 V}\left[\begin{array}{llll}
\cdots & f_{1, x} & f_{1, y} & f_{1, z} \\
\cdots & f_{2, x} & f_{2, y} & f_{2, z} \\
\cdots & f_{3, x} & f_{3, y} & f_{3, z} \\
\cdots & f_{4, x} & f_{4, y} & f_{4, z}
\end{array}\right] .
$$


The stiffness matrix of tetrahedron element is given by

$$
k=\int_{V} B^{T} C B d V .
$$

Integration in (24) is made by one-point Gaussian quadrature.The matrix of shape functions derivatives and shape functions itself $B$ is

$$
B=\left[\begin{array}{ccccccccccccc}
f_{1, x} & 0 & 0 & 0 & 0 & 0 & \ldots & f_{4, x} & 0 & 0 & 0 & 0 & 0 \\
0 & f_{1, y} & 0 & 0 & 0 & 0 & \ldots & 0 & f_{4, y} & 0 & 0 & 0 & 0 \\
0 & 0 & f_{1, z} & 0 & 0 & 0 & \ldots & 0 & 0 & f_{4, z} & 0 & 0 & 0 \\
0 & f_{1, x} & 0 & 0 & 0 & f_{1} & \ldots & 0 & f_{4, x} & 0 & 0 & 0 & f_{4} \\
f_{1, y} & 0 & 0 & 0 & 0 & -f_{1} & \ldots & f_{4, y} & 0 & 0 & 0 & 0 & -f_{4} \\
0 & 0 & f_{1, y} & f_{1} & 0 & 0 & \ldots & 0 & 0 & f_{4, y} & f_{4} & 0 & 0 \\
0 & f_{1, z} & 0 & -f_{1} & 0 & 0 & \ldots & 0 & f_{4, z} & 0 & -f_{4} & 0 & 0 \\
0 & 0 & f_{1, x} & 0 & -f_{1} & 0 & \ldots & 0 & 0 & f_{4, x} & 0 & -f_{4} & 0 \\
f_{1, z} & 0 & 0 & 0 & f_{1} & 0 & \ldots & f_{4, z} & 0 & 0 & 0 & f_{4} & 0 \\
0 & 0 & 0 & f_{1, x} & 0 & 0 & \ldots & 0 & 0 & 0 & f_{4, x} & 0 & 0 \\
0 & 0 & 0 & 0 & f_{1, y} & 0 & \ldots & 0 & 0 & 0 & 0 & f_{4, y} & 0 \\
0 & 0 & 0 & 0 & 0 & f_{1, z} & \ldots & 0 & 0 & 0 & 0 & 0 & f_{4, z} \\
0 & 0 & 0 & f_{1, y} & 0 & 0 & \ldots & 0 & 0 & 0 & f_{4, y} & 0 & 0 \\
0 & 0 & 0 & 0 & f_{1, x} & 0 & \ldots & 0 & 0 & 0 & 0 & f_{4, x} & 0 \\
0 & 0 & 0 & 0 & f_{1, z} & 0 & \ldots & 0 & 0 & 0 & 0 & f_{4, z} & 0 \\
0 & 0 & 0 & 0 & 0 & f_{1, y} & \ldots & 0 & 0 & 0 & 0 & 0 & f_{4, y} \\
0 & 0 & 0 & f_{1, z} & 0 & 0 & \ldots & 0 & 0 & 0 & f_{4, z} & 0 & 0 \\
0 & 0 & 0 & 0 & 0 & f_{1, x} & \ldots & 0 & 0 & 0 & 0 & 0 & f_{4, x}
\end{array}\right] .
$$

It is noteworthy that in contrast to the in classical theory of elasticity, the micropolar tetrahedral element is not the element of constant stress/strain. Indeed, in (25) linear shape functions appear. In (25) the following order of strain and displacement components is assumed:

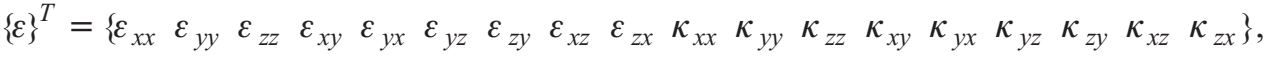

$$
\begin{aligned}
& \{u\}^{T}=\left\{\begin{array}{llllllllllllll}
u^{1} & v^{1} & w^{1} & \theta_{x}^{1} & \theta_{y}^{1} & \theta_{z}^{1} & \ldots & u^{4} & v^{4} & w^{4} & \theta_{x}^{4} & \theta_{y}^{4} & \theta_{z}^{4}
\end{array}\right\} .
\end{aligned}
$$

Constitutive matrix $C$ is defined in (11).

In order to solve various boundary value problems, a special 4-node micropolar finite element has been developed in the form of UEL (user element) procedure for commercial ABAQUS program. UEL procedure is called twice for each finite element and for each Gaussian point. In the first call, the element stiffness is welcomed. The UEL procedure calls UELMAT (user material procedure) necessary to obtain the relation between stress and strain tensors (or between stress and strain increments). Another call of UEL procedure is necessary to compute residual forces - element nodal forces resulting from element stresses - which essential in the monitoring of convergence in nonlinear problems. This second call may also require another call of UELMAT procedure. 
Linking the user element procedure with the commercial software allows one to solve large boundary value problems in an efficient way. Unfortunately for users, the visualization of the obtained results causes many problems, but it can be avoided by writing special Python scripts for ABAQUS, which create several output databases available for the postprocessor. Four output databases are necessary to show all components of strains and stresses. Note that, in micropolar elasticity, the number of strain/stress components exceeds the number of components (six) in the classical approach.

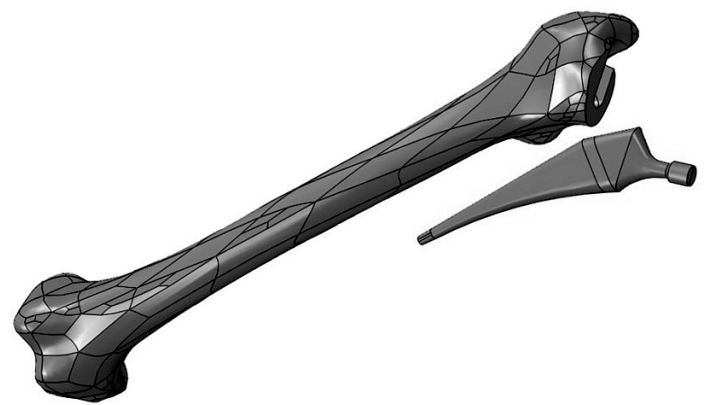

Fig. 2. Femur bone an hip implant.
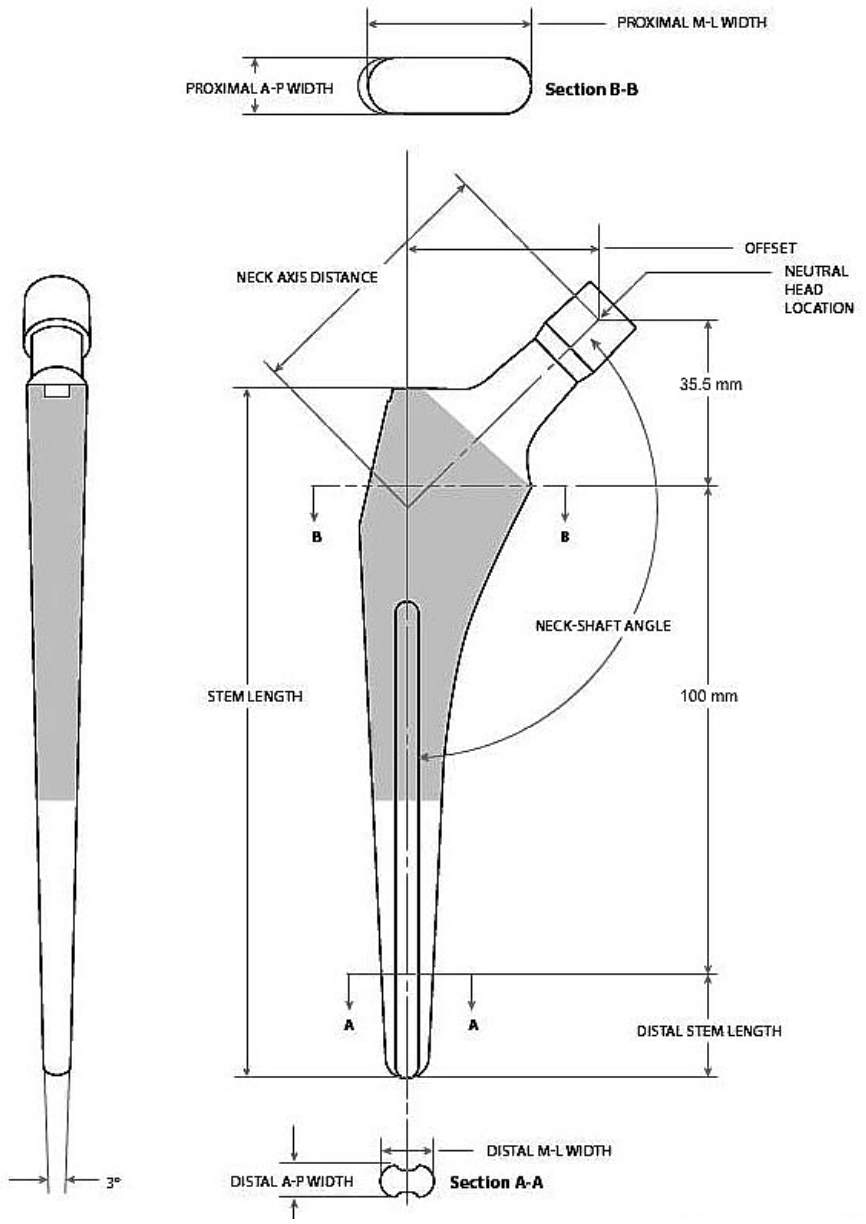

Fig 3. A400 lateralized stem specifications with parameters for different sizes (http://www.renovissurgical.com/2011/09/a400-sizes-and-specs/). 
$\mathrm{T}$ a b 1 e 2

Geometry Details for Fig. 3

\begin{tabular}{|c|c|c|c|c|c|c|c|c||}
\hline Size & $\begin{array}{c}\text { Neck } \\
\text { axis } \\
\text { distance } \\
(\mathrm{mm})\end{array}$ & $\begin{array}{c}\text { Offset } \\
(\mathrm{mm})\end{array}$ & $\begin{array}{c}\text { Stem } \\
\text { lengt } \\
h \\
(\mathrm{~mm})\end{array}$ & $\begin{array}{c}\text { Distal } \\
\text { stem } \\
\text { lengt } \\
h \\
(\mathrm{~mm})\end{array}$ & $\begin{array}{c}\text { Proximal } \\
\text { cross } \\
\text { section } \\
\text { M-L } \\
\text { width } \\
(\mathrm{mm})\end{array}$ & $\begin{array}{c}\text { Proximal } \\
\text { cross } \\
\text { section } \\
\text { A-P } \\
\text { width } \\
(\mathrm{mm})\end{array}$ & $\begin{array}{c}\text { Distal } \\
\text { cross } \\
\text { section } \\
n \text { M-L } \\
\text { width } \\
(\mathrm{mm})\end{array}$ & $\begin{array}{c}\text { Distal } \\
\text { cross } \\
\text { section } \\
n \text { A-P } \\
\text { width } \\
(\mathrm{mm})\end{array}$ \\
\hline 4.50 & 52.4 & 38.5 & 131.0 & 9.0 & 29.1 & 11.5 & 5.5 & 4.2 \\
\hline 5.25 & 52.9 & 39.3 & 132.3 & 10.5 & 29.7 & 11.8 & 6.3 & 4.5 \\
\hline 6.00 & 53.5 & 40.0 & 133.6 & 12.0 & 30.4 & 12.0 & 7.0 & 4.7 \\
\hline 6.75 & 54.0 & 40.8 & 134.9 & 13.5 & 31.0 & 12.3 & 7.8 & 5.0 \\
\hline 7.50 & 54.6 & 41.5 & 136.2 & 15.0 & 31.7 & 12.5 & 8.5 & 5.3 \\
\hline 8.25 & 55.2 & 42.5 & 137.6 & 16.5 & 32.3 & 12.8 & 9.3 & 5.6 \\
\hline 9.00 & 55.8 & 43.0 & 138.9 & 18.0 & 33.0 & 13.1 & 10.0 & 5.8 \\
\hline 9.75 & 56.3 & 43.8 & 138.9 & 19.5 & 33.7 & 13.3 & 10.8 & 6.1 \\
\hline 10.50 & 56.9 & 44.5 & 140.2 & 21.0 & 34.4 & 13.6 & 11.5 & 6.4 \\
\hline 11.20 & 57.1 & 45.3 & 141.5 & 22.5 & 35.1 & 13.8 & 12.5 & 6.6 \\
\hline 12.00 & 58.1 & 46.0 & 142.9 & 24.0 & 35.7 & 14.1 & 13.0 & 6.9 \\
\hline 13.50 & 59.3 & 47.5 & 144.1 & 27.0 & 37.0 & 14.7 & 14.5 & 7.4 \\
\hline 15.00 & 60.5 & 49.0 & 146.8 & 30.0 & 38.4 & 15.2 & 16.0 & 8.0 \\
\hline
\end{tabular}

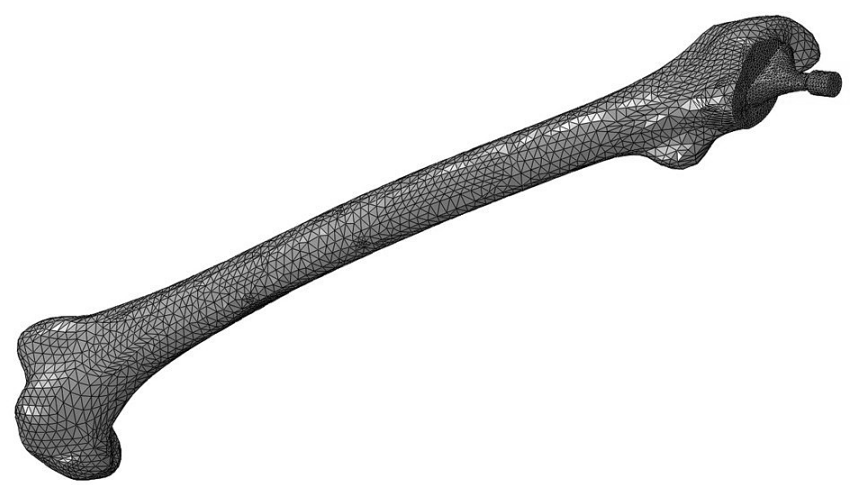

Fig. 4. FEM mesh.

Modeling of a Femur Bone with an Implant. Using the developed software we discussed earlier some test problems including also some problems for bones [34-36]. Here, as an example of real FEM modeling of a complex structure, we consider a femur bone with a hip implant (Fig. 2). A solid model of hip transplant was created using 3D CAD tools of SolidWorks 2010 (SolidWorks Corporation, USA) on the basis of A400 lateralized specification that shown in Fig. 3 and Table 2. For the bone and implant, we developed a fine mesh (Fig. 4). Here we pay a special attention to the implant area increasing there the number of elements. It was almost 100,000 elements with the maximum size of $3 \mathrm{~mm}$. The value of the static load acting on the femur is $800 \mathrm{~N}$. The load is directed along an axis extending from the upper pole of the prosthesis head to the middle 


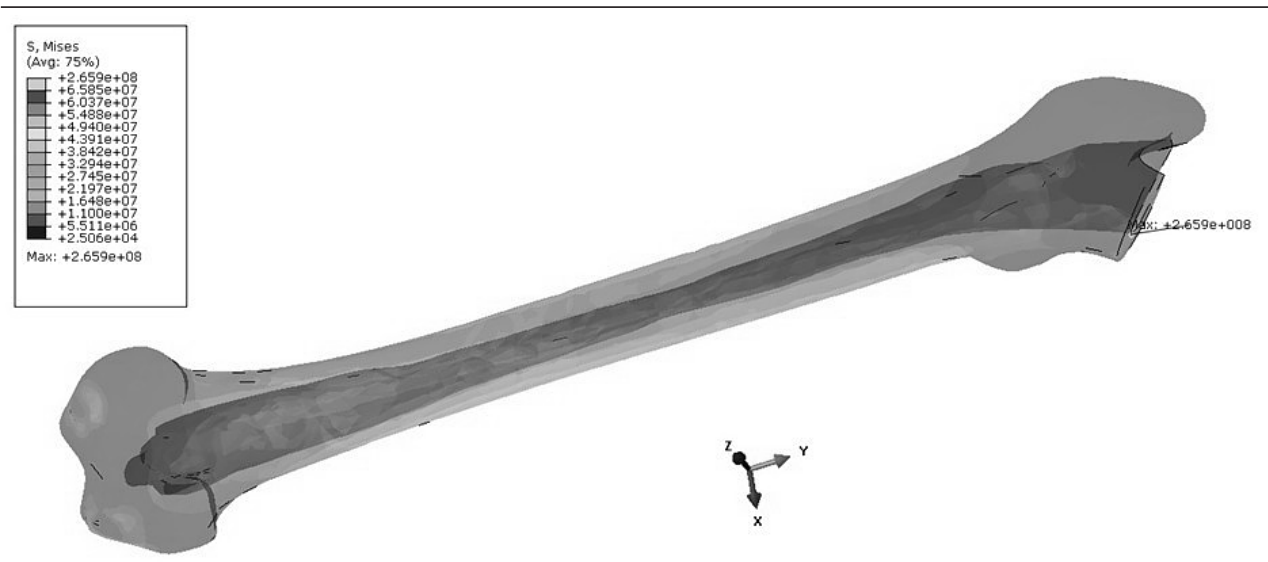

Fig. 5. Distribution of Huber-Mises stress (Pa).

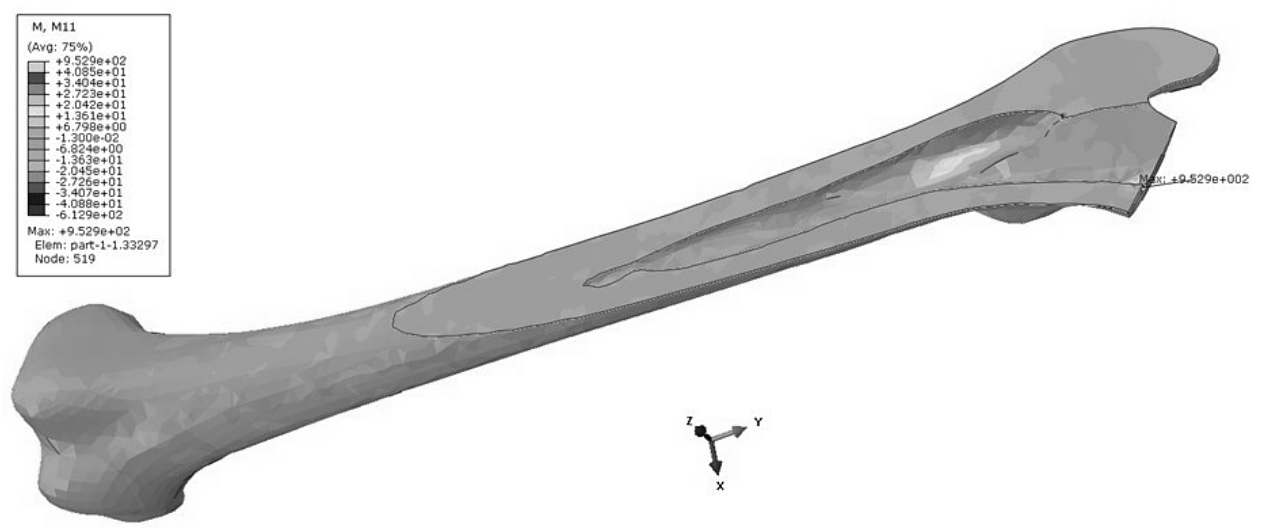

Fig. 6. Distribution of $M_{x x}$ couple stress $(\mathrm{N} / \mathrm{m})$.

of the distance between the extreme lower divisions femoral condyle. The results of calculations are given in Figs. 5 and 6. In particular, it is clear that influence of the couple stresses is important in the vicinity of some singularities such as edges of the implant (Fig. 6). This means that the rotations of the material particles and couple stresses play a role in such zones.

Conclusions. In this paper, the linear isotropic micropolar elasticity was applied to modeling of femur bones with and without implants. The analysis of bones and its interaction with implants is a complex task from the point of view of mechanics and numerical computations. Indeed, this problem combines both a complex geometry and complex material properties. A bone can be considered as a porous composite consisting of hydroxyapatite (which is mainly calcium phosphate) and collagen. For numerical analysis in addition to developed earlier hexahedral finite micropolar element we developed new specific tetrahedral micropolar finite element and implemented it the commercial software ABAQUS. The results obtained within the micropolar theory are compared with the classic linear elasticity. Numerical tests have shown that the couple stresses appear in the vicinity of singularities, such as holes and edges of the size which is comparable to the characteristic length parameters. These effects may be very important for highly porous materials such as bones and bioceramics. The presenting results may be important for personalization of the surgery. Indeed, pre-surgery planning is important to select the optimum acetabular component, and in predicting the approximate volume of components which can be used 
during the intervention. Using the developed tool one can relatively easy calculate the level of stresses, stress concentrations and other characteristics of the mechanical behavior of a bone with and without an implant.

Acknowledgments. The research leading to these results has received funding from the People Program (Marie Curie Curie ITN transfer) of the European Union's Seventh Framework Programme for research, technological development and demonstration under grant agreement No PITN-GA-2013-606878.

1. S. Cowin (Ed.), Bone Mechanics Handbook, CRC Press LLC, Boca Raton (2001).

2. Y. H. An and R. A. Draughn (Eds.), Mechanical Testing of Bone and the BoneImplant Interface, CRC Press LLC, Boca Raton (2000).

3. S. C. Cowin and D. H. Hegedus, "Bone remodeling I: theory of adaptive elasticity," $J$. Elasticity, 6, No. 3, 313-326 (1976).

4. D. H. Hegedus and S. C. Cowin, "Bone remodeling II: small strain adaptive elasticity," J. Elasticity, 6, No. 4, 337-352 (1976).

5. R. S. Lakes and J. F. Yang, "Transient study of couple stress effects in compact bone: torsion,” J. Biomech. Eng., 103, 275-279 (1981).

6. J. F. C. Yang and R. S. Lakes, "Experimental study of micropolar and couple stress elasticity in compact bone in bending," J. Biomech., 15, No 2, 91-98 (1982).

7. H. C. Park and R. S. Lakes, "Cosserat micromechanics of human bone: strain redistribution by a hydration sensitive constituent," J. Biomech., 19, No. 5, 385-397 (1986).

8. R. S. Lakes, "Experimental microelasticity of two porous solids," Int. J. Solids Struct., 22, No. 1, 55-63 (1986).

9. R. S. Lakes, "Experimental micro mechanics methods for conventional and negative Poisson's ratio cellular solids as Cosserat continua," J. Eng. Mater. Technol., 113, No. 1, 148-155 (1991).

10. I. Goda, M. Assidi, S. Belouettar, and J. F. Ganghoffer, "A micropolar anisotropic constitutive model of cancellous bone from discrete homogenization," J. Mech. Behav. Biomed., 16, 87-108 (2012).

11. I. Goda, M. Assidi, and J. F. Ganghoffer, "3D elastic micropolar model of vertebral trabecular bone from lattice homogenization of the bone microstructure," Biomech. Model. Mechan., 13, No. 1, 53-83 (2014).

12. I. Goda and J. F. Ganghoffer, "Identification of couple-stress moduli of vertebral trabecular bone based on the 3D internal architectures," J. Mech. Behav. Biomed., 51, 99-118 (2015).

13. I. Goda, F. Dos Reis, and J. F. Ganghoffer, "Limit analysis of lattices based on the asymptotic homogenization method and prediction of size effects in bone plastic collapse," in: H. Altenbach and S. Forest (Eds.), Generalized Continua as Models for Classical and Advanced Materials, Springer International Publishing (2016), pp. 179211.

14. I. Goda, R. Rahouadj, J. F. Ganghoffer, et al., "3D couple-stress moduli of porous polymeric biomaterials using $\mu \mathrm{CT}$ image stack and FE characterization," Int. J. Eng. Sci., 100, 25-44 (2016).

15. F. Dell'Isola F., D. Steigmann, and A. Della Corte, "Synthesis of fibrous complex structures: Designing microstructure to deliver targeted macroscale response," Appl. Mech. Rev., 67, No. 6, 060804-060804-21 (2016). 
16. F. Dell'Isola, I. Giorgio, M. Pawlikowski, and N. L. Rizzi, "Large deformations of planar extensible beams and pantographic lattices: Heuristic homogenization, experimental and numerical examples of equilibrium," Proc. Roy. Soc. A, 472, No. 2185 (2016), DOI: 10.1098/rspa.2015.0790.

17. D. Scerrato, I. Giorgio, and N. L. Rizzi, "Three-dimensional instabilities of pantographic sheets with parabolic lattices: numerical investigations," Z. Angew. Math. Phys., 67, No. 3, Article No. 53 (2016).

18. F. Dell'Isola, I. Giorgio, M. Pawlikowski, and N. L. Rizzi, "Large deformations of planar extensible beams and pantographic lattices: heuristic homogenization, experimental and numerical examples of equilibrium," Proc. R. Soc. A, 472, No. 2185 (2016), DOI: 10.1098/rspa.2015.0790.

19. E. Turco, F. Dell'Isola, A. Cazzani, and N. L. Rizzi, "Hencky-type discrete model for pantographic structures: numerical comparison with second gradient continuum models," Z. Angew. Math. Phys., 67, No. 4, 1-28 (2016).

20. M. Cuomo, F. Dell'Isola, L. Greco, and N. L. Rizzi, "First versus second gradient energies for planar sheets with two families of inextensible fibres: Investigation on deformation boundary layers, discontinuities and geometrical instabilities," Compos. Part B - Eng., 115, 423-448 (2017).

21. L. Placidi, L. Greco, S. Bucci, et al., "A second gradient formulation for a 2D fabric sheet with inextensible fibres," Z. Angew. Math. Phys., 67, No. 5, 114 (2016), doi.org/10.1007/s00033-016-0701-8.

22. T. Lekszycki and F. Dell'Isola, "A mixture model with evolving mass densities for describing synthesis and resorption phenomena in bones reconstructed with bioresorbable materials," Z. Angew. Math. Mech., 92, No. 6, 426-444 (2012).

23. I. Giorgio, U. Andreaus, D. Scerrato, and F. Dell'Isola, "A visco-poroelastic model of functional adaptation in bones reconstructed with bio-resorbable materials," Biomech. Model. Mechan., 15, No. 5, 1325-1343 (2016).

24. I. Giorgio, U. Andreaus, D. Scerrato, and P. Braidotti, "Modeling of a non-local stimulus for bone remodeling process under cyclic load: Application to a dental implant using a bioresorbable porous material," Math. Mech. Solids (2016), DOI: $10.1177 / 1081286516644867$.

25. I. Giorgio, U. Andreaus, T. Lekszycki, and A. Della Corte, "The influence of different geometries of matrix/scaffold on the remodeling process of a bone and bio-resorbable material mixture with voids," Math. Mech. Solids (2015), DOI: 10.1177/ 1081286515616052.

26. G. Mishuris, "Models of an interaction between two elastic media one of which is weakened by a symmetrical angular cut," Vestn. Leningrad Univ. Math., 62-66 (1985).

27. E. Radi, "Effects of characteristic material lengths on mode III crack propagation in couple stress elastic-plastic materials," Int. J. Plasticity, 23, No. 8, 1439-1456 (2007).

28. E. Radi, "On the effects of the characteristic lengths in bending and torsion on Mode III crack in couple stress elasticity," Int. J. Solids Struct., 45, No. 10, 3033-3058 (2008).

29. A. Piccolroaz, G. Mishuris, and E. Radi, "Mode III interfacial crack in the presence of couple-stress elastic materials," Eng. Fract. Mech., 80, 60-71 (2012).

30. G. Mishuris, A. Piccolroaz, and E. Radi, "Steady-state propagation of a Mode III crack in couple stress elastic materials," Int. J. Eng. Sci., 61, 112-128 (2012). 
31. L. Morini, A. Piccolroaz, G. Mishuris, and E. Radi, "On fracture criteria for dynamic crack propagation in elastic materials with couple stresses," Int. J. Eng. Sci., 71, 45-61 (2013).

32. L. Morini, A. Piccolroaz, and G. Mishuris, "Dynamic energy release rate in couplestress elasticity," J. Phys. Conf. Ser., 451, No. 1, 012014 (2013).

33. L. Morini, A. Piccolroaz, and G. Mishuris, "Remarks on the energy release rate for an antiplane moving crack in couple stress elasticity," Int. J. Solids Struct., 51, No. 18, 3087-3100 (2014).

34. V. A. Eremeyev, A. Skrzat, and F. Stachowicz, "On finite element computations of contact problems in micropolar elasticity," Adv. Mater. Sci. Eng., Article ID 9675604, 1-9 (2016).

35. V. A. Eremeyev, A. Skrzat, and A. Vinakurava, "Application of the micropolar theory to the strength analysis of bioceramic materials for bone reconstruction," Strength Mater., 48, No. 4, 573-582 (2016).

36. V. A. Eremeyev, A. Skrzat, and F. Stachowicz, "On FEM evaluation of stress concentration in micropolar elastic materials," Nanomech. Sci. Technol., 7, No. 4, 297-304 (2016).

37. E. Cosserat and F. Cosserat, Théorie des Corps Déformables, Herman et Fils, Paris (1909).

38. A. C. Eringen, Microcontinuum Field Theories: I. Foundations and Solids, Springer Science\&Business Media, New York (1999).

39. V. A. Eremeyev, L. P. Lebedev, and H. Altenbach, Foundations of Micropolar Mechanics, Springer Science\&Business Media, Berlin (2013).

40. W. Pietraszkiewicz and V. A. W Eremeyev, "On natural strain measures of the non-linear micropolar continuum,” Int. J. Solids Struct., 46, No. 3, 774-787 (2009).

41. W. Pietraszkiewicz and V. A. Eremeyev, "On vectorially parameterized natural strain measures of the non-linear Cosserat continuum," Int. J. Solids Struct., 46, No. 11, 2477-2480 (2009).

42. V. A. Eremeyev and W. Pietraszkiewicz, "Material symmetry group of the non-linear polar-elastic continuum," Int. J. Solids Struct., 49, No. 14, 1993-2005 (2012).

43. V. A. Eremeyev and W. Pietraszkiewicz, "Material symmetry group and constitutive equations of micropolar anisotropic elastic solids," Math. Mech. Solids, 21, No. 2, 210-221 (2016).

Received 01. 09. 2017 